\title{
External stimulus control of target-directed attack and biting elicited by tailshock
}

\author{
STEPHEN F. DAVIS, JAMES W. VOORHEES, and MARY NELL TRAVIS-NEIDEFFER \\ Austin Peay State University, Clarksville, Tennessee 37040
}

\begin{abstract}
Two experiments evaluating the effects of external stimuli on attack and biting in rats elicited by electric tailshock are reported. In the first experiment, stimuli presented to four groups of test subjects consisted of a stimulus animal, a stimulus animal plus taped vocalizations of a rat experiencing shock, an inanimate object, and an inanimate object plus taped vocalizations. Subjects in a fifth (control) group were tested in the absence of these stimuli. The presence of another animal in the test situation significantly increased the amount of target-directed responding. A decrease in responding, rather than increase, was shown by the subjects tested under the stimulus-object plus taped-vocalization conditions. Experiment 2 investigated the salient features of the stimulus animal and found a combination of both olfactory and visual cues to be most effective in eliciting target-directed responding. These studies indicate that the amount of target-directed attack and biting shown in this situation may be related causally to the type of sensory input received by the test animal.
\end{abstract}

Several authors (e.g., Cahoon, 1972; Moyer, 1968; Scott, 1966) have indicated that aggression is not a unitary concept. According to the system of classification developed by Moyer (1968), the application of aversive stimulation results in "irritable aggression." It was further noted by Moyer that "the most extreme form of irritable aggression is exemplified by destructive 'uncontrollable rage' directed against either animate or inanimate objects"' (Moyer, 1968, p. 72). This description would appear well suited for the research (discussed below) involving the application of electric footshock to pairs or groups of rats or electric tailshock to single, restrained rats.

An early publication by O'Kelly and Steckle (1939) stimulated considerable research on shock-induced fighting or aggression. In their procedure, six rats enclosed in a testing chamber were administered electric footshock, with subsequent aggressive or fighting responses being observed and recorded. These responses included assumption of an upright posture, teeth baring, and slapping or striking other subjects. Subsequent investigators (e.g., Ulrich \& Azrin, 1962) have studied such reactions in pairs of animals. Ulrich and Azrin (1962) reported the effects of manipulating such variables as frequency of shock presentation, intensity of shock, duration of shock, size of testing chamber, length of testing session, and sex of subjects.

This research was supported, in part, by a Tower Fund Research Grant from Austin Peay State University to the first author. James W. Voorhees and Mary Nell Travis-Neideffer are now at Texas Christian University, Ft. Worth, Texas 76129. Requests for reprints should be addressed to Stephen F. Davis, Department of Psychology, Austin Peay State University, Clarksville, Tennessee 37040 .
The importance and role of sensory input in the paired-animal situation has received experimental attention. Flory, Ulrich, and Wolff (1965) deprived rat pairs of vision and the use of vibrissae by placing hoods on them. A marked reduction in fighting ensued. A moderate decrease in fighting was shown when one pair of animals was blinded surgically. A further decrease in fighting occurred when the vibrissae were removed from these animals. These results led Flory et al. (1965) to conclude that both vision and use of vibrissae were important in determining the rate of attack under footshock. Bugbee and Eichelman (1972) reported the effects of bulbectomy, blinding, and removal of vibrissae on shock-induced fighting. Their results indicated that blind subjects and bulbectomized subjects did not show a decrease in fighting elicited by footshock. However, in accord wih the data reported by Flory et al. (1965), a significant postoperative decrease was shown by the devibrissaed subjects. Thus, the ability of the subject to receive certain stimulus input appears to be an important factor in determining the amount of aggression shown in this situation.

Variations in external stimuli provided to an intact animal have also proved influential in determining aggressive responding. Ulrich and Azrin (1962) showed that "reflexive" fighting was elicited between paired rats, yet shock of the same intensity did not cause a single rat to attack inanimate objects such as a doll. Dolls that moved rapidly about the chamber also failed to produce attack. Such responses were elicited only when a dead rat was moved about the chamber on a stick.

The study of shock-elicited aggression has not been limited to rats (either pairs or individuals) receiving 
foot-shock in a testing chamber. Azrin, Rubin, and Hutchinson (1968) introduced a method by which a single, restrained rat, through the administration of tailshock, could be made to bite an inanimate target located in front of it.

To date, a number of variables have been causally related to the amount of attack and biting shown in this situation, e.g., REM sleep deprivation (Mollenhour, Voorhees, \& Davis, 1977), shock modality (Davis, Tramill, Voorhees, Mollenhour, \& Prytula, 1977), food deprivation (Cahoon, Crosby, Dunn, Herrin, Hill, \& McGinnis, 1971), water deprivation (Hamby \& Cahoon, 1971) and lithiumchloride-induced nausea (Bisbee \& Cahoon, 1973). Oddly, the effects of manipulations of external stimuli do not appear among the variables studied in this situation. The data reported by Bugbee and Eichelman (1972), Flory et al. (1965), and Ulrich and Azrin (1962) on the effects of sensory input in the enclosed-chamber footshock situation strongly suggest the potential importance of such cues in the single, restrained-animal situation.

\section{EXPERIMENT 1}

In the first experiment, a variety of external stimuli were employed in an attempt to ascertain what differential effects on shock-elicited aggression might be produced. The basic situations used were an inanimate object, a stimulus animal, and a control condition. For two additional groups, tape recordings of an animal vocalizing during a shock session were presented with the inanimate object and the stimulus animal. The Ulrich and Azrin (1962) data suggest that the presence of a second (stimulus) animal should increase the frequency of target-directed attack and biting. Further, since the Flory et al. (1965) data indicate a decrease in aggression resulting from the selective removal of sensory input, the addition of potentially relevant stimuli might result in an increase in target-directed responding. It might be predicted that those subjects receiving the taped vocalizations, in addition to the inanimate object or stimulus animal, would show increased levels of attack and biting.

\footnotetext{
Method

Subjects. Forty-one male, albino rats, purchased from the Holtzman Company, Madison, Wisconsin, served as subjects. All subjects were approximately 90 days old at the onset of the experiment. All animals were housed individually with water and food available continuously.

Apparatus. A rat-restraining device consisting of an opaque plastic tube, measuring $21.5 \mathrm{~cm}$ in length and $7.5 \mathrm{~cm}$ in diameter, mounted on a Plexiglas sheet was used to hold the test subject. A $1.5-\mathrm{cm}$ hole at the enclosed end of the tube allowed the subject's tail to be extended from the apparatus and secured to a wood restraining rod by means of adhesive tape. The other end of the tube was open. Two pieces of No. 14 copper wire
}

permanently attached to the rod $7 \mathrm{~cm}$ apart served as tail electrodes. Thus, when the rod was secured in place it served as both a restraint rod and as an electrode carrier. A $1.5-\mathrm{mA}$ halfwave (pulsating) dc current was used and was monitored by a Jackson (Model 665-J-2) milliammeter.

The aggression target consisted of an omnidirectional lever (Lafayette Instruments, Model 90111). The lever was lightly taped with white adhesive tape to allow the subject to get better purchase with teeth and paws. The lever was mounted on a wood platform, perpendicular to the open end of the restraining tube. When the tube was in place on the platform, the lever extended acros $\$$ the midportion of the open end of the tube. The lever was $1.5 \mathrm{~cm}$ from the tube and required a movement of $1.0 \mathrm{~cm}$ to activate an attached microswitch. Closure of the microswitch, in turn, activated a pulse former, which activated a Lafayette (Model 5707PS) impulse counter.

Five stimulus conditions were employed. Stimulus objects were placed $7.50 \mathrm{~cm}$ in front, but beyond reach, of the restrained subjects. The first (control) situation consisted of a cardboard enclosure $(35 \times 35 \times 35 \mathrm{~cm})$ surrounding the end, top, and sides of the apparatus. The second condition consisted of a plywood box $(21.1 \times 14.3 \times 16.2 \mathrm{~cm})$ which housed a stimulus rat. The inside of the box was painted flat black, and the front was barred by six brass rods placed $1.8 \mathrm{~cm}$ apart. During testing, the box and stimulus animal were placed inside the cardboard enclosure. The third condition consisted of the same plywood box and stimulus rat, but also included a previously made tape recording of the stimulus animal vocalizing during a 10-min, 1.5-mA footshock session. A Channel Master (Model PC6765) tape recorder was used for both taping and playback functions. During shock sessions requiring its use, the recorder was placed external to the distal end of the cardboard enclosure (i.e., behind the stimulus object). Perforations in the box allowed the sound to enter the enclosure. Volume and tone controls were set at an intermediate (5) level. A Realistic (Model 33-1028) music/sound level meter measured the intensity of the stimuli reaching the test animals, and indicated that the taped vocalizations ranged between 80 an $90 \mathrm{~dB}$. In the fourth conditon, a $10-\mathrm{cm}$-square white block of wood was placed inside the cardboard enclosure. The final condition consisted of the white wood block plus the tape recording.

Procedure. Prior to the experiment, 40 of the 41 subjects were assigned randomly to one of five groups $(n=8)$ : Group $C$ (control), Group SA (stimulus animal), Group SA-V (stimulus animal plus vocalization), Group IO (inanimate object), and Group IO-V (inanimate object plus vocalization). The remaining subject served as the stimulus animal for Groups SA and SA-V.

To run a trial, the test subject was secured in the restraining tube and positioned in the apparatus so that its nose was approximately $1 \mathrm{~cm}$ from the target rod. Each subject was given a 5-min habituation period in the restraining tube with the appropriate target in place. A 10-min period of shock administration immediately followed habituation. During this time, each subject was given a series of 300 -msec-duration $1.5-\mathrm{mA}$ shocks at $3-\mathrm{sec}$ intervals. Thus, each subject received a total of 200 shocks. The total number of aggressive responses was recorded for each subject. The order for running subjects was determined by a table of random numbers.

\section{Results and Discussion}

Prior to analysis, all scores were converted to $\log _{10}(\mathrm{X}+1)$ scores. Group mean responses were as follows: .96 (C), .60 (IO), .49 (IO-V), 1.53 (SA), and $.73(\mathrm{SA}-\mathrm{V})$.

Two-way analysis of variance incorporating . Stimulus Object (stimulus animal vs. inanimate object) and Vocalization (presence vs. absence) factors 
was performed on the data from Groups SA, SA-V, IO, and IO-V. The results of this analysis indicated that the Stimulus Object $[F(1,28)=7.22, p<.05]$, Vocalization $[F(1,28)=11.96, p<.01], \quad$ and Stimulus Object by Vocalization interaction $[F(1,28)$ $=19.52, p<.01]$ factors were significant. Simple main effects analyses of variance were used to probe the significant interaction, and they indicated that Group SA was significantly more aggressive than Group IO $[\mathrm{F}(1,28)=15.09, \mathrm{p}<.01]$ and Group SA-V $[F(1,28)=11.17, p<.01]$. No other significant effects were found.

Since the above analyses did not include Group C (control subjects), Dunnett's test (Kirk, 1968, pp. 94-95) was used to compare it with all other groups. The results of these comparisons indicated that Group SA was significantly $(p<.01)$ more aggressive than Group $C$, which, in turn, did not differ significantly from Groups SA-V, IO, and IO-V.

In agreement with Ulrich and Azrin (1962), the results indicate that the presence of a second, stimulus animal resulted in a significant increase in responding. The prediction that subjects tested in the presence of the taped vocalizations would also show high response levels was not supported. For example, a comparison of Groups SA and SA-V indicates that the addition of taped vocalizations significantly reduced responding. Why? Observation of subjects in Group SA-V suggested the presence of a competing response. More specifically, it was noticed that these subjects vocalized more during testing than the other subjects (with the exception of subjects in Group IO-V). The presence of the tape recording appeared to increase vocalization at the expense of target-directed responding. The reasons for this finding are not readily apparent. It may be that the test subject was, for some reason, imitating the sounds it heard. On the other hand, it may be that the sound reaching the test subject's ears was so intense as to be painful, thus eliciting the increase in vocalization.

\section{EXPERIMEN'T 2}

As indicated by Experiment 1, the presence of the stimulus animal was a significant factor in directing the aggressive response. Experiment 1 yielded relatively little information concerning the nature of the salient cues provided by the stimulus animal. Contrary to our predictions, the depressed responding shown by Groups IO-V and SA-V suggested that auditory cues may be more inhibiting than beneficial in directing responding toward the aggression target. Thus, the two most likely cues operative in this situation would appear to be olfactory and/or visual in nature. Since Ulrich and Azrin (1962) failed to observe fighting when an inanimate object (doll) was moved about in the presence of a single rat receiving footshock, it might be anticipated that olfactory cues would be more potent than visual cues. However, as suggested by the Flory et al. (1965) data, a combination of cues from both modalities could certainly prove more effective than either by itself. In an attempt to isolate these specifics, the stimulus animal in Experiment 2 was caged such that olfactory and visual cues could be presented separately or in combination.

\section{Method}

Subjects. Thirty-three 150 -day-old, male, albino rats purchased from the Holtzman Co., Madison, Wisconsin, served as subjects. All subjects were housed and maintained as in Experiment 1.

Apparatus. The restraint tube, shock system, and counter used in Experiment 1 were used in Experiment 2. A cylindrical plastic container $(19.0 \mathrm{~cm}$ tall, $15.4 \mathrm{~cm}$ in diameter) was used to contain the stimulus animal. Sixty percent of the exterior surface of the container was painted flat black. The unpainted $40 \%$ formed a vertical patch from top to bottom of the container. Thus, when the container was in place $(7.5 \mathrm{~cm}$ in front of the test animal) with the black side facing the test animal, no view of the stimulus animal was afforded. Viewing of the stimulus animal was permitted by facing the unpainted portion of the container toward the test animal. Two tight-fitting plastic lids were also used. One lid was perforated with $46.50-\mathrm{cm}$ holes and was used when olfactory cues were to be presented to the test animal. The second lid was solid except for a $.50-\mathrm{cm}$ (inside diameter) brass fitting cemented in the center. When this lid was in use, a $1.52-\mathrm{m}$ piece of rubber tubing was attached to the fitting and extended out the back of the enclosure surrounding the aggression apparatus, thus eliminating stimulus-animal olfactory cues from the testing situation.

Procedure. Prior to experimental testing, four groups $(\mathrm{n}=8)$ were formed randomly; Group $\mathrm{C}$ (no stimulus animal present), Group OL-VI (olfactory and visual cues present), Group OL (olfactory cues present), and Group VI (visual cues present). The remaining animal served as the stimulus animal.

Shock-elicited aggression sessions were administered in the same manner as in Experiment 1. The order for running subjects was determined by a table of random numbers.

For subjects in Group OL-VI, the stimulus animal was placed in the cylinder with the perforated lid. The unpainted portion of the cylinder faced test animal thus providing both olfactory and visual cues. For subjects in Group OL, the cylinder was rotated so the painted surface faced the test animal and presented only olfactory cues from the stimulus animal. The unpainted portion or the cylinder faced subjects in Group VI. The lid with the rubber tubing was in place and precluded olfactory cues from the stimulus animal. In an attempt to determine what effects the cylinder itself had, one-half of the animals in Group $C$ were tested with the clear portion of the empty cylinder facing them. The remaining subjects in this group were tested without the presence of the cylinder. In all cases, the appropriate placement of the cylinder and stimulus animal took place prior to placement of the test animal into the restraint tube for the habituation period.

\section{Results and Discussion}

Group mean responses were as follows: $1.61(\mathrm{C})$, 1.50 (VI), 1.63 (OL), and 1.94 (OL-VI). Prior to overall analysis, the two subgroups comprising Group $\mathrm{C}$ were compared. Because they did not differ $[\mathrm{t}(6)=.58, \mathrm{p}<.50]$, their scores were pooled for further analysis. 
One-way analysis of variance yielded a significant Groups effect $[F(3,28)=2.96, p<.05]$. The contrast effects investigated by the Newman-Keuls procedure were of direct relevance to the initial predictions. Olfactory cues were predicted to be more salient than visual cues. Therefore, Group OL should have responded more than Group VI. Because these two groups did not differ significantly, this prediction was not supported. In view of the acute olfactory and poor visual capacities of the albino rat, the lack of significant aggression differences between these two groups was surprising. It may be recalled that in Experiment 2 stimulus-animal odors emanated from the top of the container housing the stimulus animal. When the painted side of the container faced subjects in Group OL, the diffusion of odors plus the lack of visual cues may have interfered severly with the animals' ability to isolate the odor source as being directly in front of them. The comparisons of Group C with Groups OL and VI also yielded nonsignificant differences and indicate that cues from either the olfactory or visual modality alone were no more effective in increasing responding than was the control condition where no animal was present.

The finding that Group OL-VI responded significantly $(p<.05)$ more than all other groups supported the prediction that a combination of cues from both olfactory and visual modalities would prove most efficient in increasing target-directed responding. This is in accord with the Ulrich and Azrin (1962) and Flory et al. (1965) studies. Fighting was shown by single, nonrestrained subjects in the Ulrich and Azrin (1962) study only when a dead rat (providing olfactory and visual cues) was moved about the experimental chamber. Flory et al. (1965) showed that a combination of sensory modalities was more effective in modulating aggression than a single modality.

A comparison of Experiments 1 and 2 indicates that more aggression was shown in Experiment 2 than in Experiment 1. Since previous studies (e.g., Mollenhour, Voorhees, \& Davis, 1977) have reported finding aggression differences between experiments, this finding was not totally unexpected. Concerning causal factors involved, one might want to consider; (1) differences in external stimulus conditions between the two studies, and (2) age differences between the subjects used in the two experiments. Relatively young (90-day-old) subjects were used in Experiment 1, while older (150-day-old) subjects were used in Experiment 2. Hutchinson, Ulrich, and Azrin (1965) have shown that age and aggression are positively related, suggesting that age differences may well have been the prime contributor to theses differences.

\section{REFERENCES}

Azrin, N. H., Rubin, H. B., \& Hutchinson, R. R. Biting attack by rats in response to aversive shock. Journal of the Expenmental Analysis of Behavior, 1968, 11, 633-639.

Bisbee, D. S. P., "CAHOON, D. D. The effects of induced nausea upon shock-elicited aggression. Bulletin of the Psychonomic Society, 1973, 1, 19-21.

Bugbee, N. M., \& Erchelman, B. S. Sensory alterations and aggressive behavior in the rat. Physiology \& Behavior, $1972,8,981-985$.

Cahoon. D. D. A behavioristic analysis of aggression. Psychological Record, 1972, 22. 463-476.

Cahoon, D. D., Crosey, R. M., Dunn, S., Herrin, M. S., Hilz, C. C., \& MCGinnts, M. The effect of food deprivation on shock-elicited aggression in rats. Psychonomic Science, 1971, 22, 43-44.

Davis, S. F., Tramill, J. L., Voorhees, J. W., Mollenhour, M. N., \& Prytula, R. E. Shock-elicited aggression as a function of shock modality. Bulletin of the Psychonomic Society, $1977,9,145-147$.

Flory, R. K., Ulrich, R., \& Wolff, P. C. The effects of visual impairment on aggressive behavior. Psychological Record, $1965,15,185-190$.

HaMBY, W., \& CaHoon, D. D. The effect of water deprivation upon shock elicited aggression in the white rat. Psychonomic Science, 1971, 23, 52.

Hutchinson, R. R., Ulrich, R. E., \& AzRin, N. H. Effects of age and related factors on the pain-elicited reaction. Journal of Comparative and Physiological Psychology, 1965, 59, 365-369.

KIRK, R. E. Experimental design: Procedures for the behavioral sciences. Belmont, California: Brooks/Cole, 1968.

Mollenhour, M. N., Voorhees, J. W., \& Davis, S. F. Sleepy and hostile: The effects of REM sleep deprivation on shock-elicited aggression. Animal Learning \& Behavior, 1977, 5, 148-152.

MOYER, K. E. Kinds of aggression and their physiological basis. Communications in Behavioral Biology, 1968, 2 , 65-87.

O'Kelly, L. I., \& STeckee, L. C. A note on Jong enduring emotional responses in the rat. Journal of Psychology, 1939 , 8, 125-131.

Scort, J. P. Agonistic behavior of rats and mice: A review. American Zoologist, 1966, 6, 683-701.

UlRich, R. E., \& Azrin, N. H. Reflexive fighting in response to aversive stimulation. Journal of the Experimental Analysis of Behavior, 1962, 5, 511-520.

(Received for publication June 10, 1977; revision accepted July $13,1978$. ) 